\title{
UNE FICHE PHYTOSOCIOLOGIQUE
}

\author{
par
}

\author{
A. R. Pinto Da Silva et M. MYRe (*)
}

L'idée d'employer une fiche pour les relevés d'associations nous est venue de la préoccupation de rendre les recherches plus commodes, de faciliter le travail de classement et de systématisation des éléments d'étude. Il nous semble qu'une telle fiche pourrait être particulièrement utile dans la phase de travail précédant l'étude monographique d'une certaine association par son tableau d'association, c'est à dire, lorsqu'il s'agit de faire l'inventaire des groupements d'une certaine contrée.

Nous avons cru que la fiche pourrait remplacer avantageusement le cahier de notes qu'on emploie d'ordinaire.

La fiche que nous allons décrire, après deux années d'usage, appliquée à quelques centaines de relevés s'est montrée convenable et parfaitement adaptable aux cas les plus divers.

Notre fiche phytosociologique, en carton mince, a les dimensions internationales de 5" $x$ 8".

Le recto (Fig. 1) est ainsi destiné: le coin gauche supérieur à la systématique de l'association (a), à la caractérisation physionomique de la formation (Formaçâo) et au degré de recouvrement de la strate supérieure (Exp. horiz.) ; le coin droit supérieur, à l'indication de la localité, de la division administrative et s'il est nécessaire, des coordonnées géographiques (Loc.). Au dessous suivent le numéro d'ordre du relevé (N), la date (194..), les références photographiques (Fotos) et les signatures des observateurs (Rubr.); dans la marge droite, de haut en bas, sont indiquées la surface étudiée (Area : en $\mathrm{m}^{2}$ ), l'exposition, la pente (Exp.) et l'altitude (m.s.m.); ensuite, les caractéristiques pédologiques d'ordre général (Solo), les références aux échantillons du sol recueillis (Ref.) et la formation géologique (Form. geol.), suivie des notes sur le pH, l'humidité et les carbonates, aux diverses profondeurs ( $\mathrm{pH}$; Hum.; C0. $)$. On réserve une grande surface à petits carrés pour quelques autres observations, ce qui permet la construction de graphiques, et la prise de croquis.

Le verso (Fig. 2) est destiné à l'inscription du cortège floristique (Elementos florísticos), des degrés d'abondance-dominance $(\mathrm{Q}=\mathrm{Ab}$. + Do.), sociabilité (Soc.), de la phénologie (Fen.) et d'autres indications qui se montrent nécessaires en certains cas, comme, par exemple, le degré de vitalité des espèces, la „Bonität" des arbres ou des indications sur le diamètre ou l'âge de ceux-ci (les deux dernières colonnes, à droite, ont été prévues pour ces cas). Les colonnes, à gauche, servent pour les renseignements sur la stratification (Estr.) (indication de la hauteur moyenne de chaque strate), pour les formes biologiques ( $F$. Biol.) et pour la hauteur des individus de chaque espèce (Alt). La colonne à gauche de la bande moyenne peut être occupée par les chiffres correspondant aux échantillons à déterminer.

Lorsque le cortège floristique comprend plus de 37 espèces il faut utiliser une seconde fiche.

Un chassis spécial, en zinc à défaut d'aluminium, relativement léger (280 gr), rend assez commode l'utilisation des deux faces de la fiche (Photo 1). Ce chassis ne cache aucune partie utile de la fiche car celle-ci est entourée d'un rebord de $3 \mathrm{~mm}$ environ. Des aides-mémoires peuvent être appliqués sur les faces intérieures du porte-fiches.

Une bourse en cuir, avec courroie d'attache, sert à garder, dans ses trois divisions, le porte-fiches, les fiches déjà remplies et celles en blanc, des cartes géographiques et des cahiers de notes (Photo 2).

*) Estaçâo Agronómica Nacional, Sacavem, Portugal. 


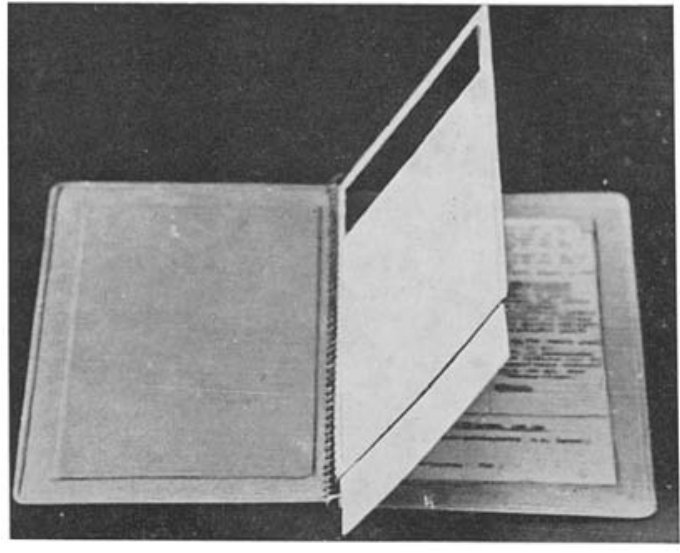

Photo 1

Chargement du chassis du porte-fiches. Le porte-fiches ouvert montrant le chassis avec la fiche placée et un aide-mémoire appliqué dans la couverture.

Remarquez la charnière en spirale permettant la superposition des couvertures quand on écrit sur la fiche.

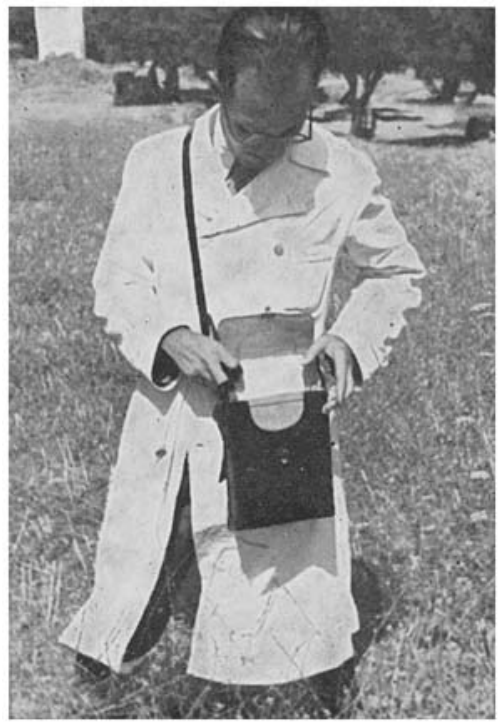

Photo 2

La fiche est gardée dans la bourse.

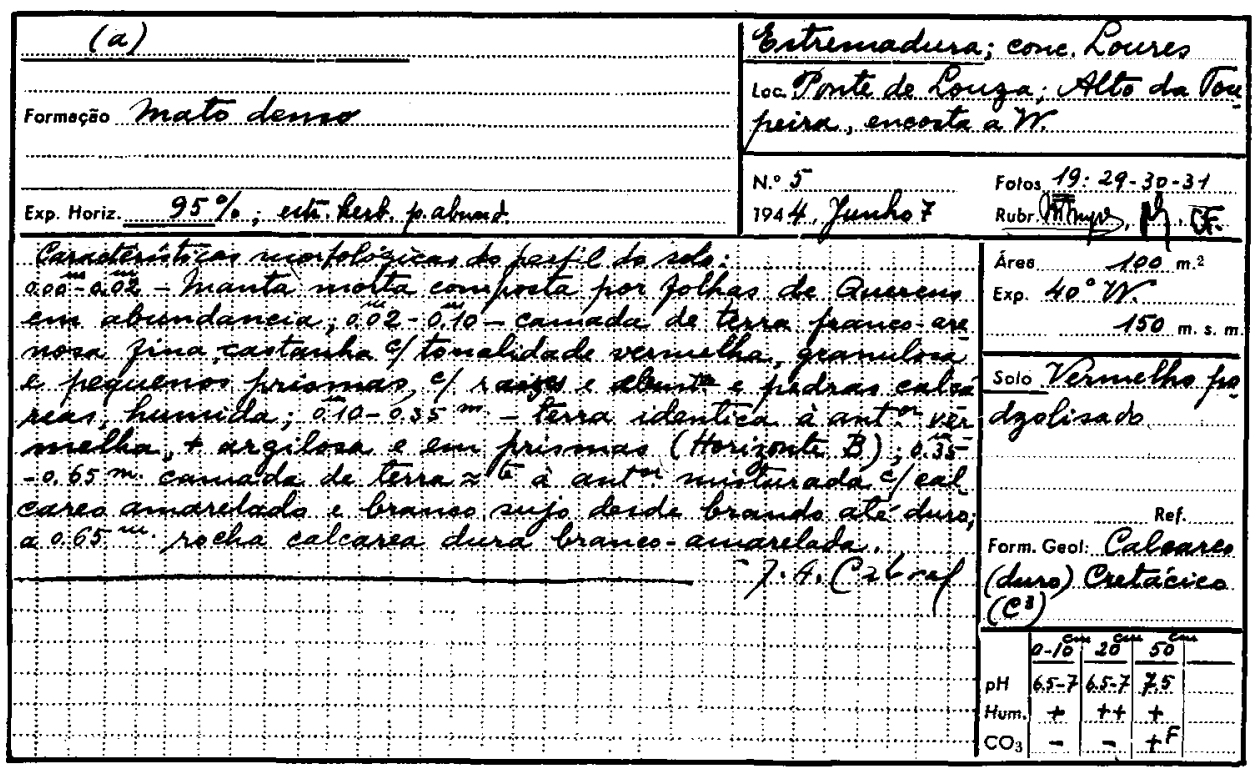

Fig. 1 - Recto de la fiche. 
Pl. V.

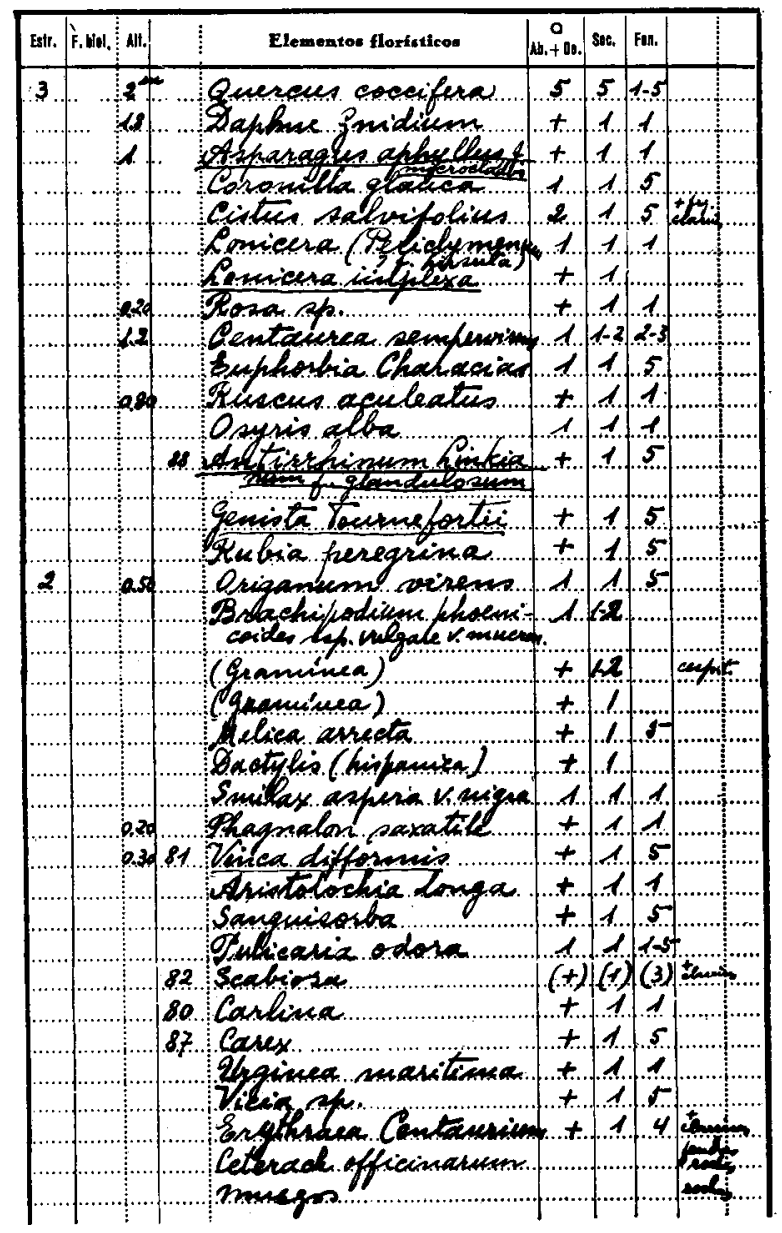

Fig. 2 - Verso de la fiche, 